\title{
ON OBSTRUCTIONS IN SPHERE BUNDLES AND IMMERSION OF MANIFOLDS
}

\author{
BY \\ J. LEVINE(1)
}

In this paper we study the following problem. Let $\xi$ be an oriented $n$-plane bundle over a complex $B$ with Euler class $X(\xi)$. If $\alpha \in H^{n}(B ; Z)$, is it possible to find another oriented $n$-plane bundle $\kappa$ stably-isomorphic to $\xi$ such that $X(\kappa)=X(\xi)+\alpha$ ? If $n$ is odd and $\alpha \neq 0$, it is well known that this impossible. If $X(\xi)=0$ we may ask a similar question about the secondary obstruction. Theorems 2 and 3 answer these questions using an operation on cohomology defined in $\S \mathrm{E}$ in terms of the indeterminancy of obstruction theory.

These results can then be applied to some problems concerned with immersing a manifold in Euclidean space. This depends upon a fundamental theorem of M. Hirsch [5]. We obtain some results on the immersibility (and imbeddabilty) of manifolds and the possible normal classes of immersions.

It is also worth mentioning that an identical investigation of nonorientable bundles can be carried out, yielding analogous results.

A. Obstruction theory. Let $\xi$ be a fibre bundle (see [13] for definitions) with base-space a complex $B$, fibre $F$ and connected structural group. This implies $\Pi_{1}(B)$ acts trivially on the homotopy groups of $F$. Let $s$ be a section of $\xi$ on $B^{r-1}(=(r-1)$-skeleton of $B)$. We denote the obstruction cocycle of $s$ by $z(s, \xi)$, an $r$-cocycle with coefficients in $\Pi_{r-1}(F)$. The obstruction class of $s$, we denote by $\zeta(s, \xi) \in H^{r}\left(B ; \Pi_{r-1}(F)\right)$. If $s_{1}, s_{2}$ are sections of $\xi$ on $B^{r}$ which agree on $B^{r-1}$, we denote the difference cochain of $s_{1}$ and $s_{2}$ by $d_{r}\left(s_{1}, s_{2}\right.$, $)$, an $r$-cochain with coefficients in $\Pi_{r}(F)$. Recall that

$$
\delta d_{r}\left(s_{1}, s_{2}, \xi\right)=z\left(s_{1}, \xi\right)-z\left(s_{2}, \xi\right)
$$

If $s_{1}$ and $s_{2}$ are extendible over $B^{r+1}, d_{r}\left(s_{1}, s_{2}, \xi\right)$ is a cocycle. We denote this difference class by $\delta_{r}\left(s_{1}, s_{2}, \xi\right)$.

If $\xi$ is an oriented $(m-1)$-sphere bundle, let $\xi_{k}$ denote the bundle of $k$-frames of $\xi$, i.e., the associated bundle whose fibre is the Stiefel manifold $V_{m, k}$. We describe $z\left(s, \xi_{k}\right)$ and $d_{r}\left(s_{1}, s_{2}, \xi_{k}\right)$. For each $r$-simplex $\sigma$ of $B$, let $v_{\sigma}$ be a section of $\xi_{m} \mid \sigma$, with proper orientation. If $x \in \partial \sigma, v_{\sigma}(x)$ induces an identification of $\xi \mid x$ with $S^{m-1}$ and $s(x)$ is identified with an element of $V_{m, k}$. If $\sigma$ is oriented this defines the

Received by the editors November 21, 1962.

(1) Partially supported by U. S. Army Research Office (Durham). 
element $z\left(s, \xi_{k}\right) \cdot \sigma \in \Pi_{r-1}\left(V_{m, k}\right)$. It is easy to see this is independent of the choice of $v_{\sigma}$. We refer to this as "framing $s \mid \partial \sigma$ with $v_{\sigma}$."

If we have sections $s_{1}$ and $s_{2}$ on $B^{r}$, agreeing on $B^{r-1}$ choose $v_{\sigma}=\left\{v_{\sigma}^{1}, \cdots, v_{\sigma}^{m}\right\}$ so that $\left\{v_{\sigma}^{1}, \cdots, v_{\sigma}^{k}\right\}=s_{2} \mid \sigma$. Then "framing $s_{1} \mid \sigma$ with $v_{\sigma}$ " defines a map $(\sigma, \partial \sigma)$ $\rightarrow\left(V_{m, k}, e\right)$, where $e$ is a base point of $V_{m, k}$. If $\sigma$ is oriented, this defines $d_{r}\left(s_{1}, s_{2}, \xi_{k}\right) \cdot \sigma \in \Pi_{r}\left(V_{m, k}\right)$; again it is independent of the choice of $v_{\sigma}$.

Finally we recall a well-known fact. Suppose $\xi$ and $\eta$ are bundles with fibres $F$ and $F^{\prime}$ with connected structural groups. Then any fibre-preserving map $\Phi: \xi \rightarrow \eta$ induces homomorphisms $\phi: \Pi_{i}(F) \rightarrow \Pi_{i}\left(F^{\prime}\right)$ (see [13]).

LEMMA 1. Let $\Phi, \phi$ be as above.

(a) If $s$ is a section of $\xi \mid B^{r-1}$ and $s^{\prime}=\Phi s$, then $\phi_{*} z(s, \xi)=z\left(s^{\prime}, \eta\right)$.

(b) If $s_{1}, s_{2}$ are sections of $\xi \mid B^{r}$ agreeing on $B^{r-1}$ and $s_{i}^{\prime}=\Phi s_{i}$, then:

$$
\phi_{*} d_{r}\left(s_{1}, s_{2}, \xi\right)=d_{r}\left(s_{1}{ }^{\prime}, s_{2}{ }^{\prime}, \eta\right) \text {. }
$$

B. Stable and nonstable isomorphisms. Let $r, t$ be integers; we define

$$
G_{r}=\lim _{k \rightarrow \infty} \Pi_{r+k}\left(S^{k}\right) ; \quad F_{r}^{t}=\lim _{k \rightarrow \infty} \Pi_{t}\left(V_{t-r+k, k}\right)
$$

as the homotopy groups in the stable range $k \geqq r+2$. If we consider the fibration $V_{t-r+k, k} \rightarrow V_{t-r+k, k-1}$ for large $k$ we have an exact sequence:

$$
F_{r}^{t} \stackrel{j}{\rightarrow} F_{r-1}^{t} \stackrel{\partial}{\rightarrow} G_{r-1} \stackrel{i}{\rightarrow} F_{r-1}^{t-1} \stackrel{j}{\rightarrow} F_{r-2}^{t-1}
$$

for $t>2 r$.

If $\xi$ is an oriented $(n-1)$-sphere bundle, we denote by $\xi^{k}$ the $k$-fold suspension of $\xi$ (the Whitney join of $\xi$ with the trivial $(k-1)$-sphere bundle); thus $\xi_{m}^{k}$ is the bundle of $m$-frames of $\xi^{k}$. Two oriented sphere-bundles $\xi, \eta$ are said to be s-isomorphic $\left(\xi \sim_{s} \eta\right)$ if $\xi^{k} \approx \eta^{k}$ (isomorphism of oriented bundles) for some $k$.

If $s$ is a section of $\xi_{k}^{k}$, the orthogonal complement of $s$ is a well-defined oriented $(n-1)$-sphere bundle; the definition is obvious. We will say two sections of $\xi_{k}^{k}$ are equivalent if there is an (orientation-preserving) automorphism of $\xi^{k}$ (covering the identity map of the base $B$ ) which carries one section into the other. It is clear that two sections of $\xi_{k}^{k}$ are equivalent if and only if their orthogonal complements are isomorphic. On the other hand if $\eta^{k} \approx \xi^{k}$, it is easy to construct a section of $\xi_{k}^{k}$ whose orthogonal complement is isomorphic to $\eta$.

If $B$ is a finite-dimensional complex, there is an integer $k$, dependent only upon $B$, with the property that any two oriented $(n-1)$-sphere bundles $\xi, \eta$ are $s$-isomorphic if and only if $\xi^{k} \approx \eta^{k}$; this is well known (see, e.g., [14]). Summarizing, we have proved:

THEOREM 1. If $B$ is a finite-dimensional complex and $\xi$ is a sphere-bundle over $B$, there is a one-one correspondence between isomorphism classes of bundles 
s-isomorphic to $\xi$ and equivalence classes of sections of $\xi_{k}^{k}$, for large enough $k$, induced by taking orthogonal complements.

Let $\xi \sim_{s} \eta$ be oriented $(n-1)$-sphere bundles such that $\xi\left|B^{r-1}=\eta\right| B^{r-1}$. We say $\alpha \in H^{r}\left(B ; F_{r-n}^{r}\right)$ is an $r$-difference of $\xi$ and $\eta$ if $\alpha=\delta_{r}\left(s_{1}, s_{2}, \xi_{k}^{k}\right)$, where $\xi, \eta$ are orthogonal complements of $s_{1}, s_{2}$, resp., satisfying $s_{1}\left|B^{r-1}=s_{2}\right| B^{r-1}$. Note that $\alpha$ is not uniquely determined by $\xi, \eta$. A representative cocycle of $\alpha$ will also be called an $r$-difference.

C. Two lemmas. Let $\xi, \eta$ be oriented $(n-1)$-sphere bundles with base space $B$, satisfying $\xi \sim_{s} \eta$ and $\xi\left|B^{r-1}=\eta\right| B^{r-1}$. Let $\alpha$ be an $r$-difference (cocycle) of $\xi$ and $\eta$, and $s$ be a section of $\left.\xi \mid B^{r-1}\left(=\eta \mid B^{r-1}\right)\right)$.

LEMMA 2. If $2 n \geqq r+3$ and $\partial: F_{r-n}^{r} \rightarrow G_{r-n}$ is the homomorphism in (2), then

$$
\partial_{*}(\alpha)=z(\xi, s)-z(\eta, s)
$$

LEMMA 3. If $2 n \geqq r+4$ and the sequence from (2):

$$
0 \rightarrow G_{r+1-n} \stackrel{i}{\rightarrow} F_{r+1-n}^{r} \stackrel{j}{\rightarrow} F_{r-n}^{r} \rightarrow 0
$$

is exact, then:

(i) $s$ is extendible over $B^{r}$ in $\xi$ if and only if it is so in $\eta$.

(ii) If $s^{\prime}, s^{\prime \prime}$ are extensions of $s$ over $B^{r}$ in $\xi$, $\eta$, resp. and $\beta$ is the Bockstein with respect to the above short exact sequence, then (regarding $\alpha$ as a cohomology class)

$$
\beta(\alpha)=\zeta\left(\xi, s^{\prime}\right)-\zeta\left(\eta, s^{\prime \prime}\right)
$$

REMARK. The restrictions on $n$ and $r$ serve only to place the coefficient groups in the stable range. Similar results hold for any integers $r, n$.

Proof of Lemma 2. Let $s_{1}, s_{2}$ be sections of $\xi_{k}^{k}$, whose orthogonal complements are isomorphic to $\xi$, $\eta$, resp., such that $s_{1}\left|B^{r-1}=s_{2}\right| B^{r-1}$. For each $r$-simplex $\sigma$, let $v_{\sigma}=\left(v_{\sigma}^{1}, \cdots, v_{\sigma}^{n+k}\right)$ and $w_{\sigma}=\left(w_{\sigma}^{1}, \cdots, w_{\sigma}^{n+k}\right)$ be properly oriented sections of $\xi_{n+k}^{k} \mid \sigma$, satisfying:

$$
\left(v_{\sigma}^{1}, \cdots, v_{\sigma}^{k}\right)=s_{1}\left|\sigma, \quad\left(w_{\sigma}^{1}, \cdots, w_{\sigma}^{k}\right)=s_{2}\right| \sigma .
$$

"Framing $s_{2} \mid \sigma$ with $v_{\sigma}$ " defines $f:(\sigma, \partial \sigma) \rightarrow\left(V_{n+k, k}, e\right)$ representing $\alpha \cdot \sigma$; "framing $\left(w_{\sigma}^{1}, \cdots, w_{\sigma}^{k+1}\right)$ with $v_{\sigma}$ " defines $g$ satisfying the following commutative diagram:

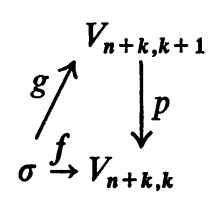


where $p$ is a projection on the initial $k$-frame. It follows from the definition of $\partial$ that $\partial(\alpha \cdot \sigma)=\left(\partial_{*} \alpha\right) \cdot \sigma$ is represented by "framing $w_{\sigma}^{k+1} \mid \partial \sigma$ with $\left(v_{\sigma}^{k+1}, \cdots, v_{\sigma}^{k+n}\right)$ ".

By identifying $\xi, \eta$ with the orthogonal complements of $s_{1}, s_{2}$, we see that $z(s, \xi) \cdot \sigma$ (resp. $z(s, \eta) \cdot \sigma)$ is represented by "framing $s \mid \partial \sigma$ with $\left(v_{\sigma}^{k+1}, \cdots, v_{\sigma}^{n+k}\right)$ (resp. $\left.\left(w_{\sigma}^{k+1}, \cdots, w_{\sigma}^{n+k}\right)\right)$. Now Lemma 2 follows easily.

Proof of Lemma 3. Statement (i) follows from Lemma 2, since $\partial=0$. Let $s_{1}^{\prime}, s_{2}^{\prime}$ be sections of $\xi_{k+1}^{k}$ induced by $\left(s_{1}, s^{\prime}\right)$ and $\left(s_{2}, s^{\prime \prime}\right)$, resp. If $p$ is as defined in the proof of Lemma 2, then from Lemma 1 we have

$$
p_{*} d_{r}\left(s_{1}^{\prime}, s_{2}^{\prime}, \xi_{k+1}^{k}\right)=\alpha .
$$

By (1), $\delta d_{r}\left(s_{1}^{\prime}, s_{2}^{\prime}, \xi_{k+1}^{k}\right)=z\left(s_{1}^{\prime}, \xi_{k+1}^{k}\right)-z\left(s_{2}^{\prime}, \xi_{k+1}^{k}\right)$, and, again by Lemma 1:

$$
i_{\#}\left(z\left(s^{\prime}, \xi\right)-z\left(s^{\prime \prime}, \eta\right)\right)=z\left(s_{1}^{\prime}, \xi_{k+1}^{k}\right)-z\left(s_{2}^{\prime}, \xi_{k+1}^{k}\right) ;
$$

now Lemma 3 follows from the definition of $\beta$.

D. Secondary obstructions. Let $\eta \geqq 2$ and $\xi$ an oriented $(n-1)$-sphere bundle. The Euler class $X(\xi) \in H^{n}(B ; Z)$ is the first obstruction to the existence of a section. If $n \geqq 4$ and $X(\xi)=0$, let $\alpha(\xi) \subset H^{n+1}\left(B ; Z_{2}\right)$ be the collection of obstructions to extending sections on $B_{n}$. If we define:

$$
h(\xi): H^{n-1}(B ; Z) \rightarrow H^{n+1}\left(B ; Z_{2}\right)
$$

by $h(\xi) \cdot \alpha=S q^{2} \alpha+w_{2}(\xi) \cdot \alpha$, it is known that $\alpha(\xi)$ is a coset of $I(\xi)=$ Image $h(\xi)$.

If $r \geqq 3$ we can similarly define $X\left(\xi_{r}\right) \in H^{n+1-r}\left(B ; F_{0}^{n-r}\right)$ and, if $X\left(\xi_{r}\right)=0$, $\alpha\left(\xi_{r}\right) \subset H^{n+2-r}\left(B ; F_{1}^{n-r+1}\right)$. We define, for $n>r+3$ :

$$
h_{r}(\xi): H^{n-r}\left(B ; F_{0}^{n-r}\right) \rightarrow H^{n+2-r}\left(B ; F_{1}^{n-r+1}\right)
$$

bv $h_{r}(\xi) \cdot \alpha=i_{*}\left(S q^{2} \alpha+w_{2}(\xi) \cdot \alpha\right)$, where $i: G_{1} \rightarrow F_{1}^{n-r}$ is the homomorphism in (2). Note that $h_{r}(\xi)=h_{r+k}\left(\xi^{k}\right)$ for any $k$.

LEMMA 4. With notation as in the preceding paragraph, $\alpha\left(\xi_{r}\right)$ is a coset of $I_{r}(\xi)=$ Image $h_{r}(\xi)$. More explicitly, if $s_{1}$ and $s_{2}$ are sections of $\xi_{r} \mid B^{n+1-r}$ agreeing on $B^{n-1-r}$, then, if $\alpha=\delta_{n-r}\left(s_{1}, s_{2}, \xi_{r}\right)$ :

$$
\zeta\left(s_{1}, \xi_{r}\right)-\zeta\left(s_{2}, \xi_{r}\right)=h_{r}(\xi) \cdot \alpha .
$$

Proof. If $B$ is a differentiable manifold and $\xi$ is its tangent bundle, this is proved in [1]. In the general case, let $M$ be a differentiable manifold containing $B^{2 n}$ as a deformation retract (at least up to homotopy type) whose tangent bundle $\tau$ satisfies $\tau \mid B^{2 n} \approx \xi^{n}$. The existence of $M$ is established in [2]. The natural map $\xi_{r} \rightarrow \xi_{n+r}^{n}$ induces section $s_{i}^{\prime}$ from $s_{i}$. Since $\tau_{n+r} \mid B^{2 n} \approx \xi_{n+r}^{n}$, it follows from [1] that:

$$
\zeta\left(s_{1}^{\prime}, \xi_{n+r}^{n}\right)-\zeta\left(s_{2}^{\prime}, \xi_{n+r}^{n}\right)=h_{n+r}\left(\xi^{n}\right) \cdot \delta_{n-r}\left(s_{1}^{\prime}, s_{2}^{\prime}, \xi_{n+r}^{r}\right) .
$$

But by Lemma $1, \zeta\left(s_{i}, \xi_{r}\right)=\zeta\left(s_{i}^{\prime}, \xi_{n+r}^{n}\right)$ and $\alpha=\delta_{n-r}\left(s_{1}^{\prime}, s_{2}^{\prime}, \xi_{n+r}^{r}\right)$. Thus Lemma 4 is proved. 
E. Some operations on cohomology. Let $\xi$ be an oriented $(n-1)$-sphere bundle over a finite-dimensional complex $B$; if $i>0$ and $r \geqq n>0$, we define partial multi-valued operations:

$$
\Phi_{r-n}^{i}(\xi): H^{r}\left(B ; F_{r-n}^{r}\right) \rightarrow H^{r+i}\left(B ; F_{r+i-1-n}^{r+i-1}\right)
$$

which will have the following properties:

LEMMA 5. (a) $\Phi_{k}^{i+1}(\xi)$ is defined on $\operatorname{Ker} \Phi_{k}^{i}(\xi)=\left\{\alpha \mid \Phi_{k}^{i}(\xi) \cdot \alpha\right.$ contains 0$\}$.

(b) $\Phi_{k}^{1}(\xi)=0$, defined everywhere.

(c) $\Phi_{k}^{2}(\xi)$ is single-valued; $\Phi_{0}^{2}(\xi)=h_{0}(\xi)$ if $n>3$.

The $\Phi_{k}^{i}(\xi)$ will be useful to us because of the following lemma. Let $K_{k}(\xi)$ $=\lim _{i \rightarrow \infty} \operatorname{Ker} \Phi_{k}^{i}(\xi)$.

LEMMA 6. Let $\xi$ be an oriented $(n-1)$-sphere bundle. If $\alpha \in H^{r}\left(B ; F_{r-n}^{r}\right)$, there exists a bundle $\eta \sim_{s} \xi$, satisfying $\xi\left|B^{r-1}=\eta\right| B^{r-1}$, with $r$-difference $\alpha$ if and only if $\alpha \in K_{r-n}(\xi)$.

We define $\Phi_{r-n}^{i}(\xi)$ as follows. Let $s$ be a section of $\xi_{k}^{k}$ (for large $k$ ) whose orthogonal complement is isomorphic to $\xi$. Given $\alpha \in H^{r}\left(B ; F_{r-n}^{r}\right), \Phi_{r-n}^{i}(\xi) \cdot \alpha$ is defined if there is a section $s^{\prime}$ of $\xi_{k}^{k} \mid B^{r+i-1}$ which agrees with $s$ on $B^{r-1}$, such that $\alpha=\delta_{r}\left(s, s^{\prime}, \xi_{k}^{k}\right)$. We define $\Phi_{r-n}^{i}(\xi) \cdot \alpha$ to be the set of $\zeta\left(s^{\prime}, \xi_{k}^{k}\right)$, for all such $s^{\prime}$. It follows immediately that:

(3) There is a section $s^{\prime}$ of $\xi_{k}^{k} \mid B^{r+i}$ which agrees with $s$ on $B^{r-1}$, such that $\alpha=\delta_{r}\left(s, s^{\prime}, \xi_{k}^{k}\right)$, if and only if $\alpha \in \operatorname{Ker} \Phi_{r-n}^{i}(\xi)$.

This implies (a) of Lemma 5. Statement (b) follows directly from the definition and (1). To prove (c), let $s^{\prime}, s^{\prime \prime}$ be sections of $\xi_{k}^{k} \mid B^{r+1}$ which agree with $s$ on $B^{r-1}$, such that $\delta_{r}\left(s^{\prime}, s^{\prime \prime}, \xi_{k}^{k}\right)$ is a coboundary. Then $s^{\prime}$ is homotopic to $s^{\prime \prime}$ on $B^{r}$ and $\zeta\left(s^{\prime}, \xi_{k}^{k}\right)=\zeta\left(s^{\prime \prime}, \xi_{k}^{k}\right)$. The second part of (c) follows from Lemma 4 .

Lemma 6 follows directly from Theorem 1 and (3), taking $i>\operatorname{dim} B$.

F. The main theorems. As usual, let $\xi$ be an oriented $(n-1)$-sphere bundle over a finite-dimensional base $B$, with Euler class $X(\xi)$ and, if $X(\xi)=0$, secondary obstruction $\alpha(\xi)$. The following theorems measure the dependence of $X(\xi)$ and $\alpha(\xi)$ on the stable class of $\xi$.

THEOREM 2. If $n \geqq 2$ is even, $\alpha \in H^{n}(B ; Z)$, there is an $\eta \sim_{s} \xi$ with $X(\eta)=X(\xi)+\alpha$ if and only if $\alpha \in 2 K_{0}(\xi)$.

THEOREM 3. If $n \geqq 4, X(\xi)=0$ and $\alpha \in H^{n+1}\left(B ; Z_{2}\right) / I(\xi)$ then there is $\eta \sim_{s} \xi$ with $\alpha(\eta)=\alpha(\xi)+\alpha$ :

(a) for $n \equiv 1$ or $2 \bmod 4$, if and only if $\alpha=0$,

(b) for $n \equiv 3 \bmod 4$, if and only if $\alpha \in r S q^{1} K_{0}(\xi)$,

(c) for $n \equiv 0 \bmod 4$, if $\alpha \in r f_{*} K_{1}(\xi)$,

where $r: H^{n+1}\left(B ; Z_{2}\right) \rightarrow H^{n+1}\left(B ; Z_{2}\right) / I(\xi)$ is the quotient map and $f: Z_{2}+Z_{2} \rightarrow Z_{2}$ is nonzero. 
REMARKs. (a) If $n$ is odd, $X(\xi)$ is an integral Stiefel-Whitney class and therefore a stable invariant of $\xi$.

(b) If $n<4$, results analogous to Theorem 3 can be easily obtained.

Proof of Theorem 2. First note that, if $\xi \sim{ }_{s} \eta$, then $\xi\left|B^{n-1}=\eta\right| B^{n-1}$. If $n>2$, Theorem 2 is a direct consequence of Lemmas 2 and 6 , since $\partial: F_{0}^{n} \rightarrow G_{0}$ is multiplication by 2 for $n$ even. But if $r=n=2$, Lemma 2 is still valid, since $\Pi_{k}\left(S^{k}\right)=G_{0}$ for $k \geqq 1$; thus Theorem 2 holds for $n=2$.

Proof of Theorem 3. For (a) note that $i: G_{1} \rightarrow F_{1}^{n}$ is a split monomorphism; therefore, $i_{*}: H^{n+1}\left(B ; G_{1}\right) \rightarrow H^{n+1}\left(B ; F_{1}^{n}\right)$ is a monomorphism and so is the induced homomorphism:

$$
i_{*}^{\prime}: H^{n+1}\left(B ; G_{1}\right) / I(\xi) \rightarrow H^{n+1}\left(B ; F_{1}^{n}\right) / I_{1}(\xi)
$$

By Lemmas 1 and $4, \alpha\left(\xi_{k+1}^{k}\right)=i_{*}^{\prime} \alpha(\xi)$ and similarly for $\eta$, if $k$ is large. If $\xi^{k} \approx \eta^{k}$, since $i_{*}^{\prime}$ is a monomorphism, $\alpha(\xi)=\alpha(\eta)$.

To prove (b) and (c) recall that $\eta \sim_{s} \xi$ implies $\eta\left|B^{n-1}=\xi\right| B^{n-1}$. By Lemma 6 , the $n$-difference $\alpha^{\prime} \in K_{0}(\xi)$. If $n \equiv 3 \bmod 4$, it follows from Lemma 3 that $\alpha(\eta)-\alpha(\xi)=r S q^{1}\left(\alpha^{\prime}\right)$. If $\eta\left|B^{n}=\xi\right| B^{n}$, then the $(n+1)$-difference $\alpha^{\prime \prime} \in K_{1}(\xi)$, again by Lemma 6 . From Lemma $2, \alpha(\eta)=\alpha(\xi)$ if $n \equiv 3 \bmod 4$, and $\alpha(\eta)-\alpha(\xi)$ $=r f_{*}\left(\alpha^{\prime \prime}\right)$ if $n \equiv 0 \bmod 4$. If $\eta\left|B^{n+1}=\xi\right| B^{n+1}$, clearly $\alpha(\xi)=\alpha(\eta)$. This completes the proof of Theorem 3 .

Note that we have not obtained a complete answer if $n \equiv 0 \bmod 4$.

G. Imbeddability and immersibility of manifolds. We will now present some applications of our results as mentioned in the introduction. We will often need some restriction on the dimensions of the manifold and the Euclidean space. For this purpose we will say a pair of integers $(r+s, r)$ is proper if the homomorphism $i: G_{r} \rightarrow F_{r}^{s-1} \cdot$ is zero, for $r, s \geqq 1$.

LEMMA 7. The pair $(m, r)$ is proper if:

(i) $r=1, m \equiv 1 \bmod 4$,

(ii) $r=2, m \equiv 1$ or $2 \bmod 4$,

(iii) $r=3, m \equiv 3 \bmod 8$,

(iv) $r=4$ or 5 , any $m$.

This follows directly from considerations of the exact sequence (2) and the results of [11].

For the remainder of this paper let $M$ be a closed orientable $(q-1)$-connected differentiable $n$-manifold. All imbeddings and immersions are differentiable unless otherwise stated. Haefliger has shown in [3] that $M$ is imbeddable in $(2 n-q+1)$-space if $n>2 q$ and immersible in $(2 n-q+1)$-space if $n>2 q-2$. The following two theorems improve these results by a few dimensions with suitable restrictions on $M$ and the integers $n$ and $q$. 
The normal Stiefel-Whitney classes of $M$ will be denoted by $\bar{W}_{i}$ (in dimension $i$ ); if $i$ is odd $>1$ (resp. even or $i=1)$, it is an integral (resp. mod 2) cohomology class. If $\xi$ is the normal bundle of an immersion, $X(\xi)$ will be referred to as the normal class of the immersion. If $X(\xi)=0, \alpha(\xi)$ will be the second normal class of the immersion. Let $M_{0}$ be the complement of an open disk in $M$.

THEOREM 4. Let $M$ be as above with $\bar{W}_{n-q}=0$.

(a) If $q=1$ and $n>4$, then $M$ is immersible in $(2 n-2)-s p a c e$ if and only if:

(i) $n \equiv 1 \bmod 4$ or

(ii) $\bar{W}_{2} \cdot \bar{W}_{n-2}=0$

(b) If $q>1$ and $n>2 q+2, M$ is imbeddable in $(2 n-q)$-space. If, in addition, $(n, q)$ is proper, $M$ is immersible in $(2 n-q-1)$-space.

THEOREM 5. Let $M$ be as above with $\bar{W}_{n-q}=W_{n-q-1}=0$.

(a) If $q>2, n>2 q+4$ and $M_{0}$ is immersed in $(2 n-q-1)$-space with second normal class $\alpha$, then $M$ is imbeddable in $(2 n-q-1)$-space if:

(i) $n \equiv q+1 \bmod 4$ or

(ii) $n \equiv q \bmod 4$ and $\alpha \in r S q^{1} H^{n-q-1}(M)$ or

(iii) $\alpha=0$.

(b) If $q \neq 2, n>2 q+4,(n, q+1)$ is proper and $M$ is immersed in $(2 n-q-1)$ space with second normal class $\alpha$, then $M$ is immersible in $(2 n-q-2)$-space if and only if (i), (ii) or (iii) hold.

We will also prove the following.

THEOREM 6. Suppose $(n, q+1)$ is proper and $M$ is imbeddable in $(2 n-q-1)$ space. Then, if $W_{2}=0, M$ is immersible in $(2 n-q-2)$-space.

From Theorems 3, 4 and 5 we can derive some results about projective spaces.

COROLlaRY. (a) The following imbeddings are possible:

(i) $C P^{n}$ in $(4 n-2)$-space if $n \neq$ power of two.

(ii) $Q P^{n}$ in $(8 n-4)$-space if $n \neq$ power of two.

(b) The following immersions are possible:

(i) $R P^{n}$ in $(2 n-3)$-space if $n \equiv 1 \bmod 4$.

(ii) $Q P^{n}$ in $(8 n-5)$-space if $n \neq$ power of two.

(iii) $Q P^{n}$ in (8n-6)-space if $Q P^{n}$ is imbeddable in (8n-5)-space.

(c) The following immersion is impossible: $C P^{n}$ in $(4 n-2)$-space if $n=$ power of two.

We obtain (a) and (b-ii) from Theorem 4 (b), (b-i) from Theorems 4(b) and 5(b-ii), (b-iii) from Theorem 6 and (c) from Theorem 4(a).

RbMARKs. (a) There is considerable overlap of these results with those of [4], [12] and [8]. 
(b) Assertion (c) of the corollary contradicts a result of [10].

(c) It will be clear that one might improve Theorems 4 and 5 with more information about the operations of $\S \mathrm{E}$.

H. The normal class of an immersion. In [7] R. Lashof and S. Smale propose the problem of determining which elements $\alpha$ of $H^{r}(M ; Z)$ can be realized as the normal class of an immersion of $M$ in $(n+r)$-space. If $r$ is odd, only $\bar{W}_{r}$ itself is possible; therefore we only consider even $r$. One restriction is that $\alpha$ $\equiv \bar{W}_{r} \bmod 2$; are there other restrictions?

If $r=n$, it is proved in [7] (see also [5]) that this is the only restriction,i.e. $\alpha$ may be any even element of $H^{n}(M ; Z)$. We shall prove:

TheOREM 7. Suppose $M$ is immersible in $(2 n-t)$-space. If $t<3$ and $n-t$ is even $>0$, every element $\alpha$ of $H^{n-t}(M ; Z)$ satisfying $\alpha \equiv \bar{W}_{n-t} \bmod 2$ is realizable as the normal class of an immersion.

I. Proofs of Theorems 4-7. We first prove a lemma.

LEMMA 8. Suppose $n>2 r$ and $(n, r-1)$ is proper. If :

(i) $M_{0}$ is imbedded in $(2 n-r)$-space or,

(ii) $M$ is immersed in $(2 n-r+1)$-space with a normal field on $M_{0}$, then $M$ is immersible in $(2 n-r)$-space.

Proof. Let $f$ be an imbedding of $M_{0}$ in $(2 n-r)$-space. By "adding a cap" we may extend $f$ to a topological imbedding of $M$ in $(2 n-r+1)$-space. According to [3] there is a differentiable imbedding of $M$ in $(2 n-r+1)$-space which agrees with $f$ outside some open disk. Clearly this reduces us to (ii).

Let $s$ be a section of $\xi \mid M_{0}$, where $\xi$ is the normal bundle of an immersion of $M$ in $(2 n-r+1)$-space. Let $s^{\prime}$ be the induced section of $\xi_{k+1}^{k} \mid M_{0}$, for large $k$. Then, by Lemma $1, \zeta\left(s^{\prime}, \xi_{k+1}^{k}\right)=i_{*} \zeta(s, \xi)$, where $i: G_{r-1} \rightarrow F_{r-1}^{n-r}$; but $i_{*}=0$ if $(n, r-1)$ is proper. Therefore $\xi_{k+1}^{k}$ has a section. We now appeal to the following result of M. Hirsch, contained in [5].

(4) If any $n$-manifold $V$ is immersed in $(n+k+1)$-space, where $k>0$, with normal bundle $\xi$, then $V$ is immersible in $(n+k)$-space if and only if there exists $\eta \sim_{s} \xi$ with a section.

This completes the proof of Lemma 8 .

Proof of Theorem 4. According to [4], $M$ is imbeddable in (2n-1)-space. If $\xi$ is the normal bundle $\alpha(\xi)$ is the single element $\bar{W}_{2} \cdot \bar{W}_{n-2}$, by [9]. Since $S q^{1}$ is zero on $H^{n-1}\left(M ; Z_{2}\right)$ and $K_{1}(\xi)=H^{n}\left(M ; Z_{2}\right)$, (a) follows from Theorem 3 and (4).

To prove (b) we first note that $M_{0}$ can be immersed in $(2 n-q-1)$-space. In fact if $M_{0}$ is immersed in $(2 n-q-1+k)$-space, the first obstruction to a normal $k$-field is $\bar{W}_{n-q}=0$ and all higher obstructions are in zero groups; we then apply 
(4). By [6] $M_{0}$ can be imbedded in $(2 n-q-1)$-space if $q>1$. Now (b) follows by "adding a cap" and smoothing (as in Lemma 8) and by Lemma 8.

Proof of Theorem 5. Suppose $M_{0}$ is immersed in $(2 n-q-1)$-space with normal bundle $\xi$ and $\alpha=\alpha(\xi)$. Now $K_{1}(\xi)=H^{n-q}\left(M ; Z_{2}\right)$ and $K_{0}(\xi)$ $=H^{n-q-1}(M ; Z)$ by Lemma 5 and $\alpha(\xi)$ is the only obstruction to a section. By Theorem 3 and (4), $M_{0}$ is immersible in $(2 n-q-2)$-space if and only if (i), (ii) or (iii) hold. If $q>2, M_{0}$ can be imbedded in $(2 n-q-2)$-space by [6]. Now (a) follows by "adding a cap" and smoothing and (b) follows from Lemma 8 for $q>2$.

Suppose $q=1$ and $M$ is immersed in (2n-2)-space with normal bundle $\xi$ and $\alpha=\alpha(\xi)$. By Theorem 3 and (4), there is an immersion of $M$ in $(2 n-2)$-space with normal field on $M_{0}$ if and only if (i), (ii) or (iii) hold, since $K_{0}(\xi)=H^{n-2}(M ; Z)$ and $K_{1}(\xi)=H^{n-1}\left(M ; Z_{2}\right)$ by Lemma 5 , and $\alpha(\xi)$ is the only obstruction to a normal field on $M_{0}$. Now (b) follows for $q=1$ by Lemma 8 .

Proof of Theorem 6. Suppose $M$ is imbedded in $(2 n-q-1)$-space with normal bundle $\xi$. Let $N$ be the total space of the sphere-bundle $\xi$. The following facts about the cohomology of $N$ is due to Massey (see [9]). There is a subring $A$ of $H^{*}\left(N ; Z_{2}\right)$, closed under cohomology operations, which is disjoint from $p^{*} H^{*}\left(M ; Z_{2}\right)$, where $p: N \rightarrow M$ is the bundle projection. Furthermore, there is an element $a \in A$ and $\alpha \in \alpha(\xi)$ satisfying:

$$
S q^{2}(a)=p^{*}(\alpha)+a \cdot p^{*}\left(\bar{W}_{2}\right) .
$$

If $\bar{W}_{2}=0$, then $p^{*}(\alpha)=0$; since $p^{*}$ is a monomorphism, $\alpha=0$. Therefore $\xi \mid M_{0}$ has a section and Theorem 6 follows from Lemma 8.

Proof of Theorem 7. Suppose $M$ is immersed in $(2 n-t)$-space with normal bundle $\xi$. It follows from Lemma 5 that $K_{0}(\xi)=H^{n-t}(M ; Z)$, if $t<3$, since $h_{0}(\xi)=0$. Now Theorem 7 follows from Theorem 2 and (4).

\section{BIBLIOGRAPHY}

1. V. Boltyanskii, Homotopy theory of continuous mappings and of vector fields, Amer. Math. Soc. Transl. 7 (1957), 135-321.

2. E. Brown, Nonexistence of low dimension relations between Stiefel-Whitney classes, Trans. Amer. Math. Soc. 104 (1962), 374-382.

3. A. Haefliger, Plongements différentiables de variétés dans variétés, Comment. Math. Helv. 36 (1961), 47-82.

4. A. Haefliger and M. Hirsch, Existence and classification of differentiable imbeddings (to appear).

5. M. Hirsch, Immersions of manifolds, Trans. Amer. Math. Soc. 93 (1959), 242-276.

6. - On imbedding differentiable manifolds in Euclidean space, Ann. of Math. (2) 73 (1961), 566-571.

7. R. Lashof and S. Smale, On immersions of manifolds in Euclidean space, Ann. of Math. (2) 68 (1958), 562-583. 
8. M. Mahowald, On the immersibility and imbeddability of the real projective spaces in Euclidean space (to appear).

9. W. Massey, Normal vector fields on manifolds, Proc. Amer. Math. Soc. 12 (1961), 33-40.

10. S. Novikov, On imbedding simply-connected manifolds in Euclidean space, Soviet Math.

Dokl. 2 (1961), 718-720.

11. G. Paechter, On the groups $\pi_{r}\left(V_{n, m}\right)$ ). I, Quart. J. Math. Oxford Ser. 7(1956), 249-268.

12. B. Sanderson, Immersions and embeddings of projective spaces, Proc. London Math. Soc. (to appear).

13. N. Steenrod, Topology of fibre bundles, Princeton Univ. Press, Princeton, N.J., 1951.

14. J. Milnor, Notes on differential topology, (mimeographed), Princeton University, Princeton, N. J., 1959.

MASSACHUSETTS INSTITUTE OF TECHNOLOGY,

Cambridge, Massachusetts 